\title{
A NOVEL TIE POINT BASED STRATEGY FOR POINT CLOUD AND IMAGERY DATA FINE REGISTRATION
}

\author{
M. Eslami ${ }^{1, *}$, M. Saadatseresht ${ }^{2}$ \\ ${ }^{1}$ School of Surveying and Geospatial Engineering, College of Engineering, University of Tehran, Tehran, Iran - meslami@ut.ac.ir \\ ${ }^{2}$ School of Surveying and Geospatial Engineering, College of Engineering, University of Tehran, Tehran, Iran - msaadat@ut.ac.ir
}

\section{Commission VI, WG VI/4}

KEY WORDS: Fine Calibration, Point Cloud, Photogrammetric Images, Coarse to Fine Registration, Terrestrial Laser Scanner, Close-Range Imagery

\begin{abstract}
:
Laser scanner generated point cloud and photogrammetric imagery are complimentary data for many applications and services. Misalignment between imagery and point cloud data is a common problem, which causes to inaccurate products and procedures. In this paper, a novel strategy is proposed for coarse to fine registration between close-range imagery and terrestrial laser scanner point cloud data. In such a case, tie points are extracted and matched from photogrammetric imagery and preprocessing is applied on generated tie points to eliminate non-robust ones. At that time, for every tie point, two neighbor pixels are selected and matched in all overlapped images. After that, coarse interior orientation parameters (IOPs) and exterior orientation parameters (EOPs) of the images are employed to reconstruct object space points of the tie point and its two neighbor pixels. Then, corresponding nearest points to the object space photogrammetric points are estimated in the point cloud data. Estimated three point cloud points are used to calculate a plane and its normal vector. Theoretically, every object space tie point should be located on the aforementioned plane, which is used as conditional equation alongside the collinearity equation to fine register the photogrammetric imagery network. Attained root mean square error (RMSE) results on check points, have been shown less than 2.3 pixels, which shows the accuracy, completeness and robustness of the proposed method.
\end{abstract}

\section{INTRODUCTION}

Photogrammetry and computer vision are the main data providers for municipalities, mapping systems, urban services. Images are widely employed to generate $2 \mathrm{D} / 3 \mathrm{D}$ information for decades to the various applications: 3D modelling (Remondino and El-Hakim 2006), urban object detection (Eslami and Mohammadzadeh 2017), building extraction (Dornaika et al. 2016) and so on.

Laser scanners [Aerial, terrestrial and mobile mapping] are the other source of the data providers, which are directly gathering the multi reflected point cloud, intensity images. Many methods have been developed to process the point cloud, as 3D building roof reconstruction (Salehi and Mohammadzadeh 2017), objects facet 3D modelling (Xu et al. 2018), point cloud segmentation and classification (Grilli, Menna, and Remondino 2017; Runceanu et al. 2017).

Plenty of research proves that imagery and point cloud datasets are complimentary (Abayowa, Yilmaz, and Hardie 2015). Inaccurate calibration and estimation of the interior orientation parameters (IOPs) and exterior orientation parameters (EOPs) between imagery and point cloud datasets leads to failure of the fine registration and alignment. To overcome such a misalignment variety of methods have been proposed, which can be categorized to groups as fallow.

Statistical/area based methods: these procedures (Omidalizarandi and Neumann 2015) are looking to find maximum statistical similarity between regular form or intensity images of the point cloud data and imagery dataset . Mutual information is a common method to calculate the statistical similarity of the point cloud and imagery dataset for EOPs estimation (Parmehr et al. 2014). Coherent Differences between imagery and point cloud data is an important problem, which makes the statistical methods unusual for fine registration.

Multi-view based Methods: multi-view based approaches typically convert 2D-3D alignment issue into a 3D-3D. Commonly, SFM and MVS methods are applied on imagery data and dense point cloud is formed. then, the well-known iterative closest point (ICP) method is employed to estimate fine transformation parameters between imagery and point cloud data (Yang and Chen 2015).

Feature based methods: these types of strategies are using extracted and matched point (corner and blob points) (Huang, Zheng, and $\mathrm{Hu}$ 2018; Mishra and Zhang 2012), line/edge (Barsai et al. 2017) and plane/surface (Safdarinezhad, Mokhtarzade, and Valadan Zoej 2016) as corresponding features to fine register imagery and point cloud data. Point and line feature based approaches main complexity is to find corresponding ones from imagery and point cloud data.

In this paper, a novel strategy is developed to overcome aforementioned methods imperfection. As a main motivation, we are consuming non corresponding features [points, lines and planes] between photogrammetric imagery and point cloud datasets for coarse to fine registration. In such a case, proposed strategy using tie points of the photogrammetric imagery and converting it to object space points. Theoretically, these points should be as possible as close to the point cloud data if the IOPs and EOPs of the imagery network to be correct. By considering such an assumption, conditional equation beside to the collinearity equations, enforcing object space photogrammetric points to lie on the point cloud estimated differential planes to estimate correct IOPs and EOPs of the imagery data.

\footnotetext{
* Corresponding author
} 


\section{METHODS AND MATERIALS}

We developed a novel strategy to fine register the photogrammetric imagery and laser scanner point cloud data. Figure 1, illustrates the proposed strategy.

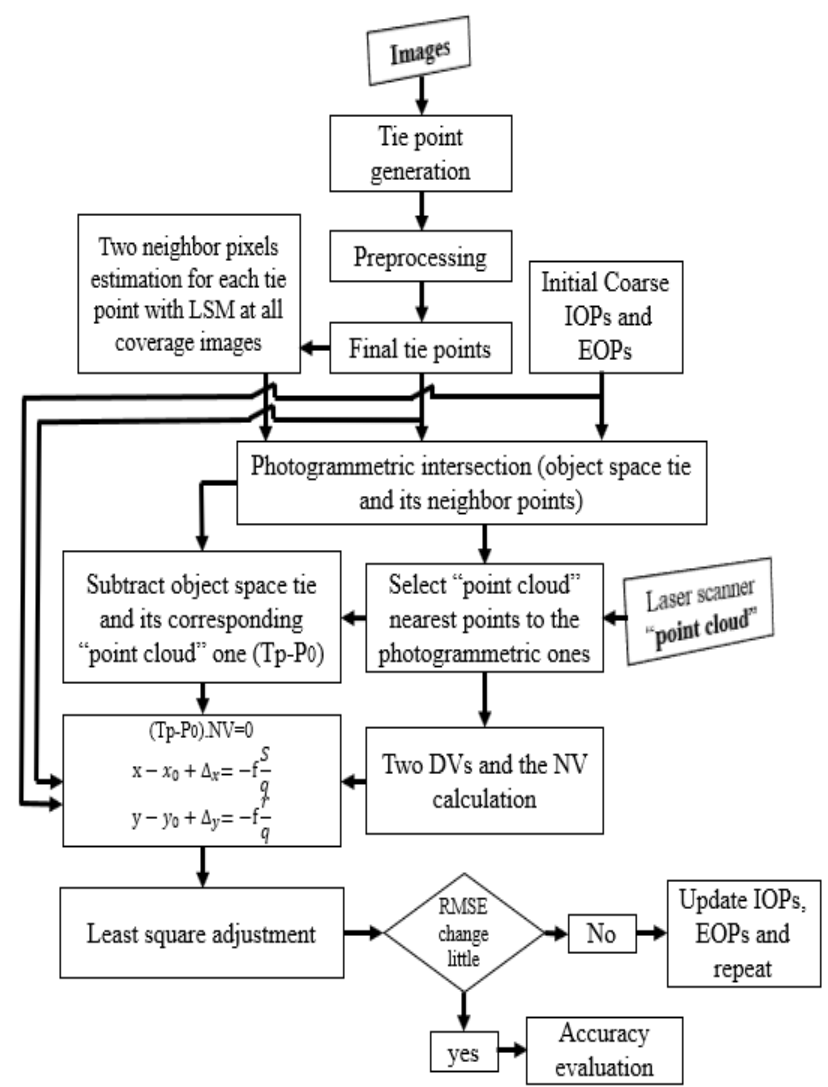

Figure 1. Flowchart of the proposed method

On the photogrammetric imageries corresponding tie points based on well-known scale-invariant feature transform (SIFT) (Lowe 2004) method are extracted and matched. Then, in the preprocessing step, two groups of the tie points are removed. first, corresponding tie points which are matched in only two images. Second, tie points which are placed in the gap area (empty of the generated points) of the point cloud dataset.

At that, for each persisted tie point, two neighbor pixels are selected and matched by least square matching (LSM) method in all coverage images, while these neighbor pixels and tie point are not located on a line. After that, initial coarse IOPs and EOPs of the images are employed to reconstruct object space coordinates of the tie point and its two neighbor pixels. The output of the method until this step is three object space photogrammetric points.

Then, nearest point to each object space photogrammetric points is estimated in the point cloud data. Estimated three point cloud points are used to calculate a plane and its normal vector (NV). There is a a fundamental geometric relationship for a plane, that the NV and directional vector (DV) will have a dot product of zero.

$$
\begin{gathered}
(\mathrm{Tp}-\mathrm{P} 0) \cdot \mathrm{NV}=0 \\
\mathrm{x}-x_{0}+\Delta_{x}=-\mathrm{f} \frac{S}{q} \\
\mathrm{y}-y_{0}+\Delta_{y}=-\mathrm{f} \frac{r}{q}
\end{gathered}
$$

Theoretically, every object space tie point should be located on the aforementioned plane (Figure 2), which is used as conditional equation (equation (1)) alongside the collinearity equation. Finally, least square adjustment (LSA) has been applied.

where $T_{P}$ and $\mathrm{P}_{0}$ are the object space tie point coordinate and its corresponding nearest point cloud point. $\mathrm{x}, \mathrm{y}$ are image coordinate of the tie point and $\mathrm{x}_{0}, \mathrm{y}_{0}, \Delta \mathrm{x}, \Delta \mathrm{y}$ and $\mathrm{f}$ are the IOPs. $\mathrm{s}, \mathrm{r}$ and $\mathrm{q}$ are the equations which containing EOPs and object space coordinate of the tie point.
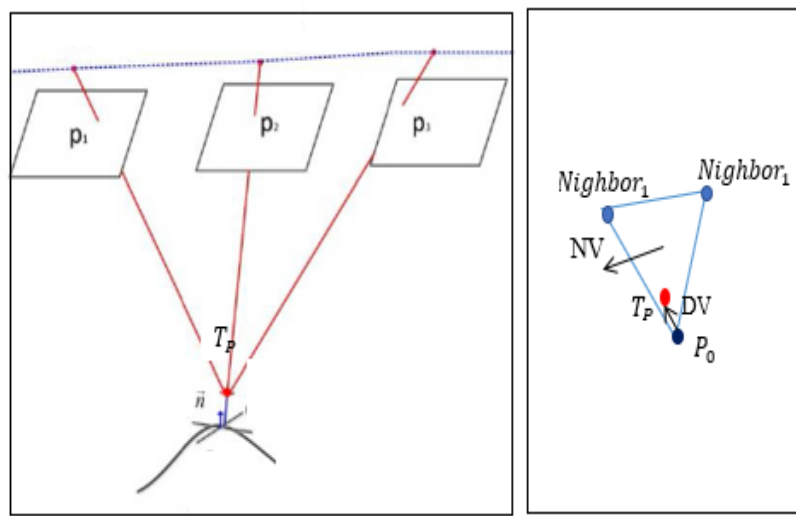

Figure 2. object space tie point and point cloud differential plane (left) and point cloud plane, DV, NV (right),

\section{IMPLEMENTATION RESULT AND DISCUSSION}

A part of our close-range laboratory of photogrammetry is captured with 14 coverage images by Canon 7D (Figure 3). Also, the data contains ScanStation2 laser scanner point cloud data. Further information about our dataset is illustrated in Table 1 .

\begin{tabular}{|c|c|c|c|c|}
\hline $\begin{array}{c}\text { Sensor } \\
\text { Type }\end{array}$ & $\begin{array}{c}\text { Distance to } \\
\text { Object }(\mathbf{m})\end{array}$ & $\begin{array}{c}\text { Focal } \\
\text { Length }(\mathbf{m m})\end{array}$ & GSD (m) & $\begin{array}{c}\text { Point } \\
\text { density }(\mathbf{m} \mathbf{2})\end{array}$ \\
\hline Camera & $4-6$ & 24 & 0.001 & - \\
\hline Laser scanner & $5-6$ & - & - & 180000 \\
\hline
\end{tabular}

Table 1. Dataset specialization

4084 initial corresponding tie points are estimated by implementation of the SIFT descriptor. A number of the tie points reduced to 1220 points by removing two image ones. Also, we removed the tie points of the point cloud gap area and final number of the corresponding tie points decreased to 921 points. Figure 4 shows the initial generated and final processed tie points.

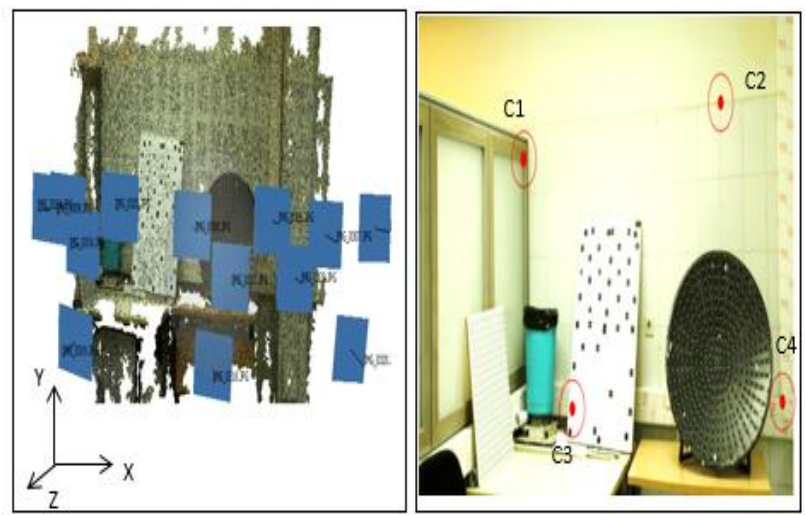

Figure 3. test scene (left) and check points location (right) 
After preprocessing step, observation equations: point to the plane conditional equation and collinearity equations for each tie point are formed and LSA is done.

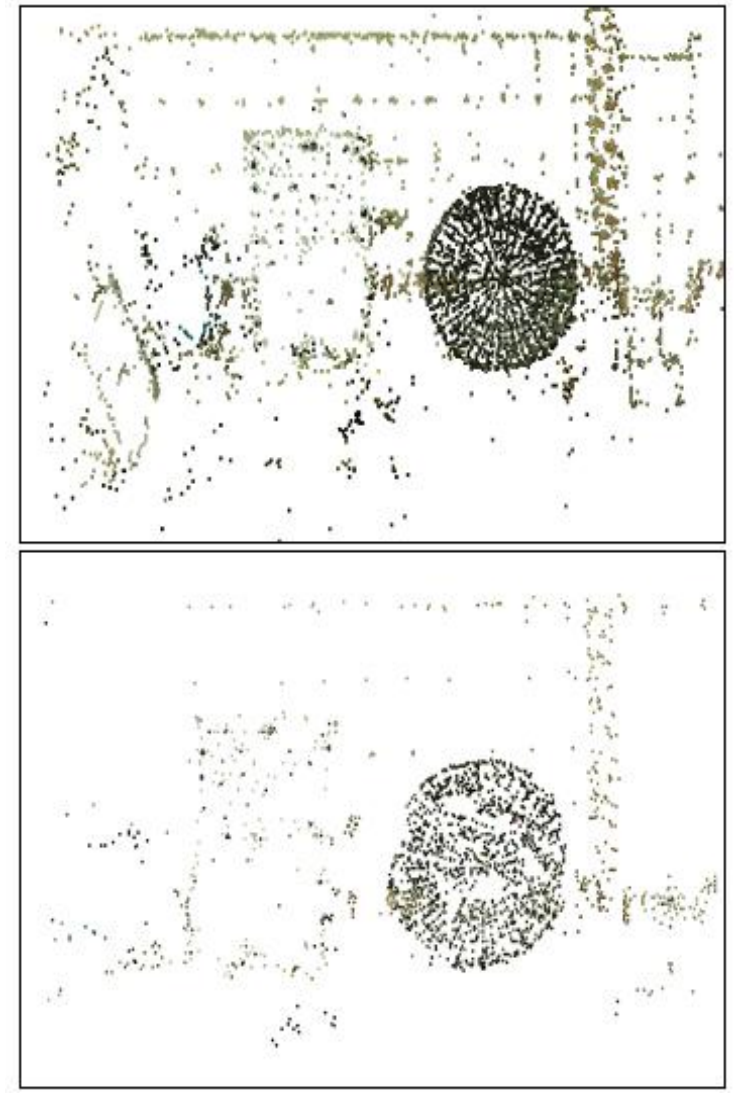

Figure 4. before (top) and after (bottom) preprocessing the corresponding tie points

Check points are used to assess the developed strategy. Four well distributed check points are shown in figure 3 (right). Selected check points are simply recognizable and identifiable in the imagery data and also in the point cloud data. Table 2 shows the average, root mean square error (RMSE) and maximum error for initial coarse and final fine registration on the check points.

\begin{tabular}{|cccc|}
\hline \multirow{2}{*}{ Error type } & \multicolumn{3}{c|}{ Coarse registration accuracy (pixel) } \\
\cline { 2 - 4 } & Maximum error & Average error & RMSE \\
\hline Total (XYZ) & 39.04 & 29.67 & 30.60 \\
\hline \multirow{3}{*}{ Error type } & \multicolumn{3}{c|}{ Fine registration accuracy (pixel) } \\
\cline { 2 - 4 } & Maximum error & Average error & RMSE \\
\hline Total (XYZ) & 2.51 & 2.15 & 2.25 \\
\hline
\end{tabular}

Table 2. average, RMSE and maximum errors before and after applying developed method

The total RMSE of the initial coarse registration is 30.60 pixels which is decreased to 2.25 pixels. Average registration accuracy decreased to 2.15 pixels. Furthermore, maximum error on the check point $\mathrm{C} 4$ equals to 2.51 pixels. As an overall result, horizontal result of the photogrammetric network is more accurate than vertical one.
A comparison between coarse and fine registration accuracies on the check points is illustrated in figure 5. Initial course registration accuracy of the all check points is larger than 19 pixels, while by applying the developed strategy the accuracy is decreased under 3 pixels. Maximum and minimum check point accuracy is decreased from 39.05 and 19.32 pixels to the value of 2.51 and 1.91 pixels respectively.

By consuming point cloud accurate and precise, the enforcement of the photogrammetric tie points to be located on the differential plane as conditional equation makes registration more accurate. The obtained results have been proved the ability of the developed strategy.

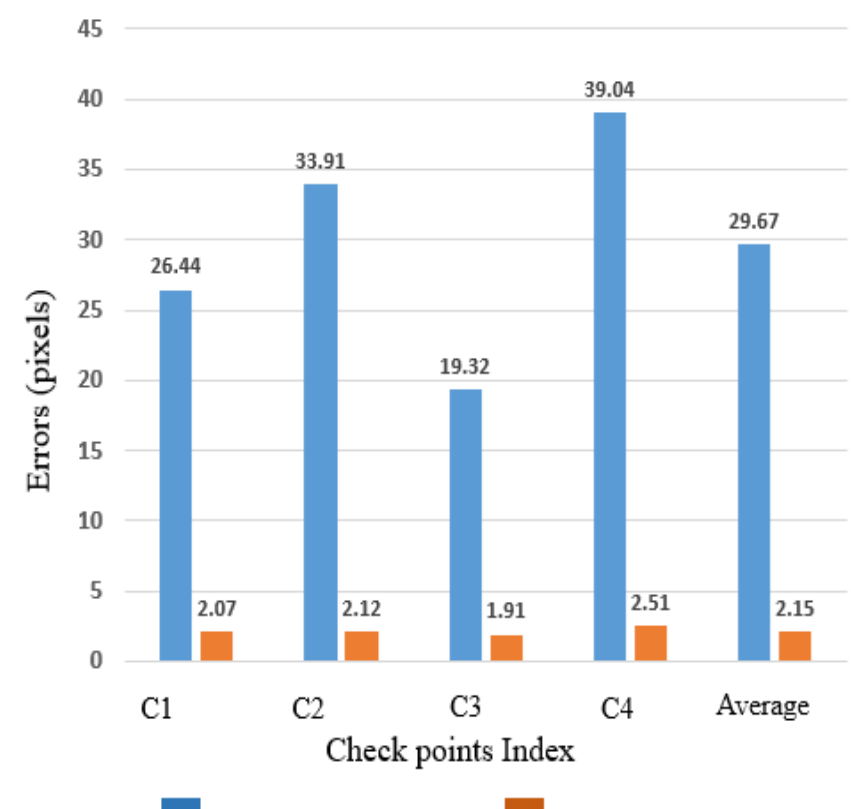

Coarse registration Fine registration

Figure 5. comparison between coarse and fine registration accuracies on the check points

\section{CONCLUSION}

Misalignment and non-accurate calibration of the imagery and point cloud datasets leads to unreliable productions and services. To address such an issue in this paper a novel feature based strategy is developed. Our method not only needs non direct corresponding features between imagery and point cloud datasets, but also employs both tie (imagery) and plane (point cloud) features.

To reach such a strategy, the tie points extracting from photogrammetric images are preprocessed and non-robust ones have been removed. After that, two neighbor pixels for each one of the tie points are estimated and by photogrammetric intersection their object space coordinates are estimated. Then, three photogrammetric points have been used to estimate nearest point cloud points. After that, three point cloud points are used to estimate a plane and NVs. Every object space tie point should be located on its corresponding point cloud differential plane. This concept is used as conditional equation beside the collinearity equation based on LSA for fine registration.

Achieved RMSE results on the four well distributed check points, have been shown less than 2.3 pixels. Such a result proves the robustness, high accuracy, automation and practicality of the developed strategy. In future works, line and 
plane features will be added to our analyzed for fine registration.

\section{REFERENCES}

Abayowa, Bernard O., Alper Yilmaz, and Russell C. Hardie. 2015. "Automatic Registration of Optical Aerial Imagery to a LiDAR Point Cloud for Generation of City Models." ISPRS Journal of Photogrammetry and Remote Sensing 106:68-81.

Barsai, Gabor, Alper Yilmaz, Sudhagar Nagarajan, and Panu Srestasathiern. 2017. "Registration of Images To Lidar and GIS Data Without Establishing Explicit Correspondences." Photogrammetric Engineering \& Remote Sensing 83(10):705-16.

Dornaika, Fadi, Abdelmalik Moujahid, Youssef El Merabet, and Yassine Ruichek. 2016. "Building Detection from Orthophotos Using a Machine Learning Approach: An Empirical Study on Image Segmentation and Descriptors." Expert Systems with Applications 58:130 42.

Eslami, Mehrdad and Ali Mohammadzadeh. 2017. "A Novel Method for Urban Land Cover Mapping Based on New Vegetation Indices and Texture-Spectral Information from Fused Visible and Hyperspectral Thermal Infrared Airborne Data." European Journal of Remote Sensing 50(1):320-31.

Grilli, E., F. Menna, and F. Remondino. 2017. "A Review of Point Clouds Segmentation and Classification Algorithms." International Archives of the Photogrammetry, Remote Sensing and Spatial Information Sciences - ISPRS Archives 42(2W3):339-44.

Huang, Rongyong, Shunyi Zheng, and Kun Hu. 2018. "Registration of Aerial Optical Images with LiDAR Data Using the Closest Point Principle and Collinearity Equations." Sensors 18(6):1770.

Lowe, David G. 2004. "Distinctive Image Features from ScaleInvariant Keypoints." International Journal of Computer Vision 60(2):91-110.

Mishra, Rakesh and Yun Zhang. 2012. "A Review of Optical Imagery and Airborne Lidar Data Registration Methods." The Open Remote Sensing Journal 5(1):54-63.

Omidalizarandi, Mohammad and Ingo Neumann. 2015. "Comparison of Target-and Mutual Informaton Based Calibration of Terrestrial Laser Scanner and Digital Camera for Deformation Monitoring." The International Archives of Photogrammetry, Remote Sensing and Spatial Information Sciences 40(1):559.

Parmehr, Ebadat G., Clive S. Fraser, Chunsun Zhang, and Joseph Leach. 2014. "Automatic Registration of Optical Imagery with 3D LiDAR Data Using Statistical Similarity." ISPRS Journal of Photogrammetry and Remote Sensing 88:28-40.

Remondino, Fabio and Sabry El-Hakim. 2006. "Image-Based 3D Modelling: A Review." The Photogrammetric Record 21(115):269-91.

Runceanu, Lavinia S., Susanne Becker, Norbert Haala, and Dieter Fritsch. 2017. "Indoor Point Cloud Segmentation for Automatic Object Interpretation." 147-59.

Safdarinezhad, Alireza, Mehdi Mokhtarzade, and Mohammad Javad Valadan Zoej. 2016. "Shadow-Based Hierarchical Matching for the Automatic Registration of Airborne LiDAR Data and Space Imagery." Remote Sensing 8(6):466.

Salehi, A. and A. Mohammadzadeh. 2017. "Building Roof Reconstruction Based on Residue Anomaly Analysis and Shape Descriptors from Lidar and Optical Data."
Photogrammetric Engineering \& Remote Sensing 83(4):281-91.

$\mathrm{Xu}$, Yusheng, Wei Yao, Sebastian Tuttas, Ludwig Hoegner, and Uwe Stilla. 2018. "Unsupervised Segmentation of Point Clouds from Buildings Using Hierarchical Clustering Based on Gestalt Principles." IEEE Journal of Selected Topics in Applied Earth Observations and Remote Sensing 11(11):4270-86.

Yang, Bisheng and Chi Chen. 2015. "Automatic Registration of UAV-Borne Sequent Images and LiDAR Data." ISPRS Journal of Photogrammetry and Remote Sensing 101:262-74 